\title{
On Improving Incentive in a Supply Chain: Wholesale Price Contract vs Quantity Dependent Contract
}

\author{
Ximin Huang, Sin-Man Choi and Wai-Ki Ching \\ Advanced Modeling and Applied Computing Laboratory, Department of Mathematics, \\ The University of Hong Kong, Pokfulam Road, Hong Kong. \\ E-mail: hehe1121@hkusua.hku.hk, kellyci@hkusua.hku.hk,wching@hkusua.hku.hk.
}

\begin{abstract}
In this paper, we first study the performance of a supply chain consisting of one retailer and one supplier. The supplier sets the price scheme of some goods and the retailer then decides the order level and sells the goods in the market. Specifically, a quadratic cost function is assumed here to approximate the $U$-shape cost curve commonly observed in industries. Two kinds of contracts offered by the supplier are investigated, namely wholesale price contract and quantity dependent contract. Wholesale price is fixed under the first contract but varies depending on order level under the second one. We show that certain wholesale price contract successfully induces the retailer to order at a level such that supply chain profit is maximized, but extra cost in implementation may occur due to supplier's disagreement on this price. Given this, we propose an efficiency measure to show to what extent the wholesale price contract helps to increase supply chain profit. For quantity dependent contract, we show that it can coordinate the supply chain and leads to a proportional division of supply chain profit. We then generalize the analysis to cover the case of multiple retailers and single supplier where similar results are also obtained.
\end{abstract}

Index Terms - coordination, game theory, quantity dependent contract, retailer, supply chain, wholesale price contract

\section{INTRODUCTION}

Supply chain coordination contracts have been extensively studied. They are attractive because if coordination is achieved, supply chain profit, which is to be split between retailers and suppliers, will be maximized, and hence Pareto optimality is possible. Hence coordination contracts help to solve the "double marginalization" problem, which was first identified by Splenger in [7], and lead to a win-win outcome [2], [8]. In this paper, we confine our attention to a supply chain consisting of only one supplier and one retailer first and then generalize the analysis to include the situation with multiple retailers and single supplier.

The majority of existing literature on coordination contracts consider the newsvendor's problem with stochastic demand. But here we assume that retailer can sell out all of his/her order at the market clearing price. The two kinds of contracts studied here are wholesale price contract and quantity dependent contract. Wholesale price contracts are often observed in the industries because they have a very simple form and the cost associated with such offer is very low. It can be shown that certain wholesale price contract is able to induce the retailer to order at supply chain optimal level. However, supplier may actually prefer a higher price for his/her own benefit. Hence, in order for the contract to be agreed upon by both retailer and supplier, additional transfer payment to the supplier should be expected and hence extra negotiation or administration cost occurs. To measure whether such extra effort is worthwhile, we define the efficiency of the wholesale price contract to show to what extent the contract helps to boost profit. Next we consider a quantity dependent contract. Such contract is very similar to the quantity discount contract (see for instance, [9]) that may appear in many different forms, because it specifies a price with dependence on the order level. However, it differs from the quantity discount contract that the price here needs not to be decreasing with order level. Cachon has demonstrated in [2] that proportional profit division can lead to one kind of quantity discount contract that coordinates the supply chain with arbitrary profit allocation within a fixed cost model. Similar method is used here to construct a quantity dependent contract for coordination within a quadratic cost model.

Here we assume the cost function to be quadratic to approximate a U-shape average total cost curve. U-shape average total cost curves have long been recognized in various manufacturing industries. The reason is that some fixed cost arises even before the start of production process while typically, marginal cost of production increases with production quantity due to diminishing marginal productivity. So average fixed cost keeps decreasing while marginal cost keeps increasing. At early stage of production, economy of scale is observed because as long as marginal cost remains below average fixed cost, average total cost keeps decreasing. However, as production quantity increases, marginal cost rises above average fixed cost and leads to increase of average total cost, hence a U-shape cost curve is formed. A quadratic function can give quite a good approximation of such U-shape curve. This is the rationale of setting the cost function to be quadratic.

The rest of this paper is structured as follows. Section II introduces the model and the basic settings; also, the optimal order level that maximizes the supply chain profit is shown to exist and its value is given. In Section III, we first investigate with what price a wholesale price contract can help to induce the retailer to order at the supply chain profit maximizing level; we then look into the supplier's incentive on setting the price 
and show that disagreements appear between supply chain's and supplier's choices of price; then we proceed to measure the efficiency of the wholesale price contract. After that, we apply the proportional profit devision method to derive a quantity dependent contract. In Section IV, we extend the analysis to study a supply chain with multiple retailers and single supplier where similar results are obtained. Finally, concluding remarks are given in Section V to discuss further research issues.

\section{THE Model}

In the proposed model, we assume that there is one retailer and one supplier in the supply chain. The following events happen in sequence: the supplier produces goods at some cost and sets a price scheme of the products; next, the retailer decides the quantity of his/her purchase and then be charged by the supplier according to that price scheme; finally the retailer sells all the ordered goods in the market at the market clearing price. We first show that there exists an optimal level of purchase by retailer that can maximize the profit of the supply chain as a whole. This result will be used in later sections to construct some supply chain coordination contracts.

Here we consider the average total cost of the products to be a typical U-shape curve. For ease of exposition, we model the cost function to be a convex quadratic function which serves as an approximation for such U-shape feature of the cost curve. Denote the level of purchase made by the retailer as $x$. The unit cost function is defined as:

$$
c(x)=a x^{2}-2 b x+c \quad \text { with } \quad a, b, c>0
$$

such that $c>b^{2} / a$ to guarantee that $c(x)$ is strictly convex, positive and has a unique global minimum for $x>0$. Therefore the total cost is: $x c(x)=a x^{3}-2 b x^{2}+c x$. Since the demand curve of a product is typically down-ward sloping, the market clearing price when the inventory level is $x$ can be formulated as:

$$
p(x)=A-\xi x \quad \text { with } \quad A, \xi>0 .
$$

Here the positive constant $A$ can be regarded as an indicator capturing the market condition (for example, showing the seasonal fluctuations of demand or reflecting the macro economic states), with a higher value of $A$ meaning the market is in a better condition. The reciprocal of the parameter $\xi$ can be regarded as a proxy for the price elasticity of demand of the product. Both values are assumed to be known and stay constant for the time being. Thus the revenue function is defined as:

$$
R(x)=(A-\xi x) x=A x-\xi x^{2} .
$$

We remark that $A>c$ must hold in order to ensure the supplier to get positive profit through the trade. Now the profit of the whole supply chain is given by

$$
\Pi=R(x)-x c(x)=-a x^{3}+(2 b-\xi) x^{2}+(A-c) x .
$$

The optimal order level $x^{*}$ satisfies the first order condition:

$$
\frac{\partial \Pi}{\partial x}=-3 a x^{2}+2(2 b-\xi) x+(A-c)=0
$$

and the corresponding second order condition. We have

Proposition 1: The profit of the whole supply chain attains its maximum at the optimal order quantity

$$
x^{*}=\frac{(2 b-\xi)+\sqrt{(2 b-\xi)^{2}+3 a(A-c)}}{3 a} .
$$

All proofs can be found in [4].

We note that the optimal order quantity $x^{*}$ increases with $A$. This means the optimal level of order for the supply chain as a whole is higher if market condition is better, which is consistent with intuition. Transfer payment within the supply chain will not have any impact on the total wealth. Hence the value of $x^{*}$ which maximizes supply chain profit remains unchanged.

\section{Wholesale Price Contract vs Quantity DEPENDENT CONTRACT}

In this section, we devote our effort into searching for a price scheme offered by the supplier so as to provide incentive for the retailer to place his/her order exactly at the optimal order quantity $x^{*}$, so that the whole supply chain profit can be maximized. Two kinds of contracts are looked into in this section. The first one is called wholesale price contract, which charges the retailer a fixed price for every unit purchased. The other one is called quantity dependent contract, which charges a variable per unit price depending on the order level $x$.

\section{A. Wholesale Price Contract}

Suppose the supplier sets price per unit to be identically $w$ for every item. Given this, the retailer's profit would be

$$
\Pi_{R}(w)=x(A-\xi x)-w x .
$$

In order to maximize this profit, set

$$
\frac{\partial \Pi_{R}(w)}{\partial x}=2 \xi x-(A-w)=0 .
$$

Hence the order level $x_{R}^{*}(w)$ chosen by the retailer given $w$ must be

$$
x_{R}^{*}(w)=\frac{A-w}{2 \xi} .
$$

We remark that $x_{R}^{*}(w)$ is a decreasing function in $w$, which coincides with intuition, for if the wholesale price increases, the retailer usually responses with a lower demand. If one wants to determine value of $w$ such that the order quantity decided by the retailer is also able to maximize the supply chain profit, we need $x_{R}^{*}(w)=x^{*}$. Substituting (5) and (7) in and solving the equation yields:

$$
\bar{w}=\frac{1}{3 a}\left(3 a A-4 \xi b+2 \xi^{2}-2 \xi \sqrt{(2 b-\xi)^{2}+3 a(A-c)}\right) .
$$

But for the contract to be feasible, the supplier must be earning a non-negative profit, i.e., $\bar{w} \geq c(x)$ must hold at $x=x^{*}$. We have the following proposition.

Proposition 2: In order for the supplier to earn a nonnegative profit, i.e., $\bar{w} \geq c(x)$ at $x=x^{*}$, one of the following conditions must hold:
(a) $\xi<\frac{b}{2}$
or
(b) $\xi \geq \frac{b}{2}$ and $A \geq \frac{(2 \xi-b) b}{a}+c$. 
Moreover, if none of these conditions is satisfied, i.e., $\bar{w}<$ $c\left(x^{*}\right)$, then the retailer's order is always less than the supply chain optimal $x^{*}$.

Regarding the first part of Proposition 2, recall that by construction, the reciprocal of $\xi$ can serve as a proxy for price elasticity of demand of a product. Hence the proposition suggests that for a certain kind of product, if it is elastic enough (with small $\xi$ ), the wholesale price contract is always feasible in the sense that it ensures a non-negative profit for the supplier. However, if the product has a low elasticity (with large $\xi$ ), for example, some necessities, the contract is feasible between supplier and retailer only when the market condition is good enough (with $A \geq(2 \xi-b) b / a+c$ ), because otherwise the supplier will be earning a negative profit. The second part of the proposition reveals that if the product is very inelastic and the market condition is very bad, which lead to the failure of the wholesale price contract, then the retailer will always order less than the supply chain optimal level.

1) Supplier's Choice: We have shown that certain wholesale price contract can induce the retailer to order at such a level that the supply chain profit is maximized. In the following discussion, we show that the supplier's interests do not agree with that of the supply chain in the sense that (s)he will try to set a higher wholesale price if (s)he has the right to do so. Here we first identify the optimal wholesale price set by the supplier and then define and calculate the efficiency of the wholesale price contract so as to illustrate under what conditions the wholesale price contract can improve the performance of the supply chain more significantly.

With a wholesale price $w$, the supplier's profit is given by

$$
\Pi_{S}(w)=w x-x c(x)=-a x^{3}+2 b x^{2}+(w-c) x .
$$

The order level $x_{R}^{*}(w)$ is determined by the retailer when given the value of $w$, so if we take the first order derivative of supplier's profit with respect to price at $w=\bar{w}$, we get

$$
\begin{aligned}
& \left.\quad \frac{d \Pi_{S}}{d w}\right|_{w=\bar{w}}=\left.\left(\left(-3 a x^{2}+4 b x+(w-c)\right) \frac{d x}{d w}+\frac{\partial \Pi_{S}}{\partial w}\right)\right|_{w=\bar{w}} \\
& =\left(-3 a\left(x^{*}\right)^{2}+4 b x^{*}+(\bar{w}-c)\right) \frac{d x}{d w}+x^{*}=x^{*}>0 .
\end{aligned}
$$

Hence it can be expected that at $w=\bar{w}$ and the corresponding social optimal order level $x=x^{*}$, the supplier still has the incentive to further increase the wholesale price so as to increase his/her own profit.

In what follows, the optimal wholesale price set by the supplier will be identified. To distinguish it from the results we already have, here we denote this optimal value chosen by supplier as $\overline{w_{S}}$ and the corresponding optimal order level as $x_{S}^{*}$.

Proposition 3: The wholesale price chosen by the supplier to maximize his/her own profit is

$$
\overline{w_{S}}=\frac{\left(3 a A+4 \xi^{2}-4 b \xi\right)-2 \xi \sqrt{(2 b-2 \xi)^{2}+3 a(A-c)}}{3 a} .
$$

As a result, the order level decided by the retailer in this case will be

$$
x_{S}^{*}=\frac{(2 b-2 \xi)+\sqrt{(2 b-2 \xi)^{2}+3 a(A-c)}}{3 a} .
$$

Comparing the wholesale price $\overline{w_{S}}$ and $\bar{w}$ set by the supplier and the supply chain respectively, the following conclusion can be drawn.

Proposition 4: The supplier prefers a higher wholesale price than that chosen by the supply chain, i.e., $\overline{w_{S}}>\bar{w}$.

This proposition confirms the implication from $d \Pi_{S} / d w>$ 0 at $w=\bar{w}$.

2) Efficiency of the Wholesale Price Contract: We define the efficiency of the wholesale price contract as:

$$
R=\frac{\Pi\left(\overline{w_{S}}\right)}{\Pi(\bar{w})} .
$$

It is the supply chain profit without wholesale price contract over the one with the contract. Obviously $R \in[0,1)$. Moreover, if $R$ is small, it means the wholesale price contract helps to significantly boost the profit of the supply chain when compared to the case where the contract is absent and the supplier can set the wholesale price freely. Recall that previously, we already have equations (4), (5), and (9). So it is straightforward to check that

$$
\left\{\begin{array}{l}
\Pi(\bar{w})=\frac{1}{27 a^{2}}\left(2\left(y^{2}+z\right)\left(y+\sqrt{y^{2}+z}\right)+y z\right) \\
\Pi\left(\overline{w_{S}}\right)=\frac{1}{27 a^{2}}\left(2\left((2 b+\xi) y_{S}+z\right)\left(y_{S}+\sqrt{y_{S}^{2}+z}\right)+(2 b+\xi) z\right)
\end{array}\right.
$$

with $y=2 b-\xi, y_{S}=2 b-2 \xi$ and $z=3 a(A-c)$. Hence we have

$R=\frac{\Pi\left(\overline{w_{S}}\right)}{\Pi(\bar{w})}=\frac{2\left((2 b+\xi) y_{S}+z\right)\left(y_{S}+\sqrt{y_{S}^{2}+z}\right)+(2 b+\xi) z}{2\left(y^{2}+z\right)\left(y+\sqrt{y^{2}+z}\right)+y z}$.

Proposition 5: We have the following two results for the efficiency $R$ :
(a) $\lim _{\xi \rightarrow 0} R=1$ and
(b) $\lim _{a(A-c) \rightarrow 0} R \in\left[\frac{20}{27}, 1\right]$.

We remark that the first limit shows the wholesale price contract is less urgent for more elastic products (with smaller $\xi)$, since even if the right to set the wholesale price is entitled to the supplier, the resulting supply chain profit is still quite close to the optimized one. However, when dealing with more inelastic product, the importance of the wholesale price contract becomes more significant because it helps to increase supply chain profit to a greater degree. The second limit shows that when the market condition is very bad (with smaller $A$ ), we can predict that if wholesale price contract is not put in practice, the worst case for the supply chain is that it only earns $20 / 27 \approx 74 \%$ of the amount that it could have earned with the contract. Here we exhibit some numerical examples (Fig. 1 \& Fig. 2) to show the behavior of $R$ under different sets of parameters.

Note that in these two pairs of examples, a similar phenomenon which can be observed is that: while letting $a, b$ and $c$ stay the same, the parameter of the second figure lets $a(A-c)$ to be a much smaller value, and hence $R$ displays a different behavior.

\section{B. Quantity Dependent Contract}

Suppose now the supplier charges the retailer $w(x)$ for per unit purchased, where the price $w(x)$ is depending on the 


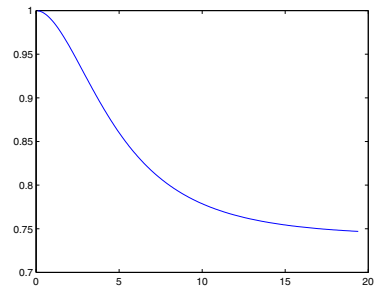

(a) $A=40$

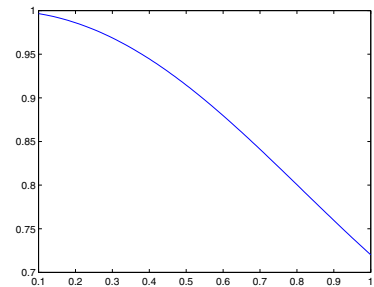

(b) $A=3.01$
Fig. 1. Dependence of $R$ on $\xi$ with $a=1, b=1, c=2$

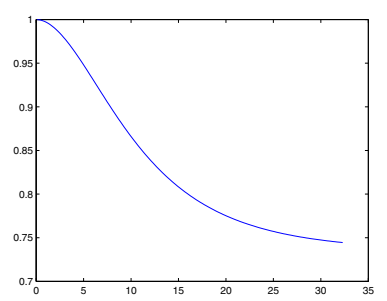

(a) $A=40$

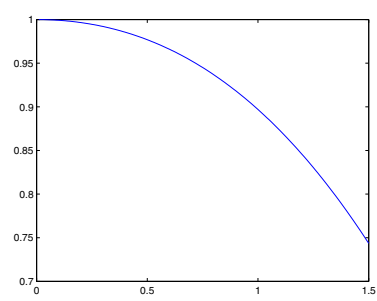

(b) $A=3.01$
Fig. 2. Dependence of $R$ on $\xi$ with $a=5, b=3, c=3$

purchase level $x$. In this case, retailer's profit becomes

$$
\Pi_{R}=(A-\xi x) x-w(x) x .
$$

In order to align interests of the retailer with that of the supply chain and hence to induce the retailer to choose the social optimal order quantity $x^{*}$, we let

$$
\Pi_{R}=\lambda \Pi \quad \text { with } \quad 0 \leq \lambda \leq 1 .
$$

Substituting (4) and (11) in and simplifying it gives:

$$
\bar{w}(x)=(1-\lambda)(A-\xi x)+\lambda\left(a x^{2}-2 b x+c\right) .
$$

In this contract $\bar{w}\left(x^{*}\right) \geq c\left(x^{*}\right)$ always holds, because for the supply chain to earn a non-negative profit (which is the sum of profits of both the supplier and retailer), we must have the inequality $A-\xi x^{*} \geq a\left(x^{*}\right)^{2}-2 b x^{*}+c$ holds. That is to say, such contract is feasible regardless of the market condition and elasticity of the product. This can be reckoned as an advantage of this quantity dependent contract over the wholesale price contract for it can be more widely used.

We can also write $\bar{w}(x)$ in another way as:

$$
\bar{w}(x)=a \lambda x^{2}-(2 b \lambda+\xi(1-\lambda)) x+A(1-\lambda)+c \lambda .
$$

So it can be seen that $\bar{w}(x)$ is actually a quadratic function of $x$. We further remark that this quantity dependent contract helps to allocate a proportion of $\lambda$ of the total supply chain profit to the retailer and a proportion of $(1-\lambda)$ to the supplier. The final division of total wealth may depend on the bargaining power of the two parties or some other factors. Similar method of imposing proportional profit devision has been used by Cachon in [2] to derive a quantity discount contract under a newsvendor's model with fixed per unit cost.

\section{Extension to Multiple Retailers}

In all previous discussions, we always assume that there is only one retailer and one supplier in the supply chain. In this section, we generalize the previous analysis to study a supply chain composed of multiple retailers and single supplier. We further assume that all the retailers are homogeneous in the sense that they all face a same market clearing price and a same wholesale price scheme. Since for the supply chain as a whole, the total revenue and total cost are unchanged, so the optimal level of order also stays the same, i.e., supply chain profit is maximized when

$$
x=x^{*}=\frac{(2 b-\xi)+\sqrt{(2 b-\xi)^{2}+3 a(A-c)}}{3 a} .
$$

Here we first give some basic settings and notations as follows. As in Section II, cost function is assumed to be quadratic, specifically: $c(x)=a x^{2}-2 b x+c$ stands for the average total cost. There are $n$ retailers $(n \geq 1)$. Ordering decisions are made by them independently to maximize their own profits, denote their order levels as $x_{1}, x_{2}, \ldots, x_{n}$ respectively and $X_{n}=\sum_{i=1}^{n} x_{i}$. For expositional simplicity, define $\overline{x_{i}}=X_{n}-x_{i}$. Here the market clearing price depends on the total orders from all retailers, so market clearing price will be given by $A-\xi X_{n}$.

\section{A. Wholesale Price Contract}

In this section we assume that the supplier charges all retailers a fixed price for per unit purchased, whose value is independent of the order levels. To distinguish it from previous results, denote the wholesale price here by $w_{n}$. For the $i$-th retailer, his/her profit will be

$$
\Pi_{i}\left(w_{n}\right)=\left[A-\xi\left(x_{i}+\overline{x_{i}}\right)\right] x_{i}-w_{n} x_{i} .
$$

In order to maximize his/her own profit, the optimal order level for the $i$-th retailer, which is denoted as $x_{i}^{*}\left(w_{n}\right)$, must be the solution to $0=\partial \Pi_{i} / \partial x_{i}=A-2 \xi x_{i}-\xi \overline{x_{i}}-w_{n}$. Solving it yields

$$
\begin{gathered}
x_{i}^{*}\left(w_{n}\right)=\frac{A-\overline{\xi \overline{x_{i}^{*}\left(w_{n}\right)}-w_{n}}}{2 \xi} \text { and it holds for all } i \text { 's. } \\
X_{n}^{*}\left(w_{n}\right)=\sum_{i=1}^{n} x_{i}^{*}\left(w_{n}\right)=n\left(\frac{A-w_{n}}{2 \xi}\right)-\frac{1}{2}(n-1) X_{n}^{*}\left(w_{n}\right) .
\end{gathered}
$$

Further simplification gives

$$
X_{n}^{*}\left(w_{n}\right)=\frac{n}{n+1}\left(\frac{A-w_{n}}{\xi}\right) .
$$

Then in order to induce the retailers to make an aggregate order at the social optimal level, we need:

$$
X_{n}^{*}\left(w_{n}\right)=x^{*}
$$

Substituting (5) and (16) in and solving the equation yields:

$\overline{w_{n}}=\frac{1}{3 a}\left[3 a A-\left(\frac{(n+1) \xi}{n}\right)\left((2 b-\xi)+\sqrt{(2 b-\xi)^{2}+3 a(A-c)}\right)\right]$ 
Proposition 6: In order to have $\overline{w_{n}} \geq c(x)$ at $x=x^{*}$, one of the following conditions must hold:

(a) $\xi<\frac{2 n}{n+3} b$ or

(b) $\xi \geq \frac{2 n}{n+3} b \quad$ and $\quad A \geq c+\frac{1}{a}\left(\frac{n+3}{n} \xi-2 b\right)\left(\frac{1}{2} b-\frac{n-1}{4 n} \xi\right)$.

Note that the following inequality holds:

$\overline{w_{n}}-\bar{w}=\frac{n-1}{3 a n} \xi\left((2 b-\xi)+\sqrt{(2 b-\xi)^{2}+3 a(A-c)}\right)>0$

Hence, when more retailers enter the market, the wholesale price that coordinates the supply chain increases while the coordinating order level remains unchanged. So the supplier's profit has in fact increased. This means, when there are more retailers, the coordination contract will be more attractive to the supplier.

1) Supplier's Choice: In the previous discussions, we identify the level of wholesale price that can coordinate the supply chain. In this subsection, we explore the supplier's own incentive on setting the wholesale price in the presence of multiple retailers. And then we will define and calculate the efficiency of the wholesale price contract. To distinguish results here from those above, denote the optimal wholesale price set by the supplier and the corresponding aggregate order level by all the retailers as $\overline{w_{n S}}$ and $X_{n S}^{*}$ respectively.

In the last section, we have seen that when given the wholesale price $w_{n}$, the retailers, by independently maximizing their own profits, decide to make an aggregate order of the amount $X_{n}^{*}\left(w_{n}\right)=\left(n\left(A-w_{n}\right)\right) /((n+1) \xi)$. Hence, when supplier is making the pricing decision, (s)he expects this. Thus the supplier's profit can be rewritten as:

$$
\begin{aligned}
& \Pi_{n S}\left(w_{n}\right) \\
& =-a\left(X_{n}^{*}\left(w_{n}\right)\right)^{3}+2 b\left(X_{n}^{*}\left(w_{n}\right)\right)^{2}+\left(w_{n S}-c\right) X_{n}^{*}\left(w_{n}\right) \\
& =-a\left(\frac{n\left(A-w_{n}\right)}{(n+1) \xi}\right)^{3}+2 b\left(\frac{n\left(A-w_{n}\right)}{(n+1) \xi}\right)^{2}+\left(w_{n}-c\right) \frac{n\left(A-w_{n}\right)}{(n+1) \xi}
\end{aligned}
$$

So as for maximization, we set $\frac{d \Pi_{n S}}{d w_{n}}=0$.

Solving the equation gives:

$$
\overline{w_{n S}}=\frac{1}{3 a}\left(3 a A-\left(\frac{n+1}{n}\right) \xi\left(y_{n S}+\sqrt{y_{n S}^{2}+z}\right)\right)
$$

with $y=2 b-\xi, y_{n S}=2 b-\frac{n+1}{n} \xi$ and $z=3 a(A-c)$. It can be easily checked that second order condition is satisfied: $\left.\left(\partial^{2} \Pi / \partial w^{2}\right)\right|_{w=\overline{w_{n S}}}=-2(n /(n+1) \xi)^{2} \sqrt{y_{n S}^{2}+z}<0$. By

$$
X_{n}^{*}\left(w_{n}\right)=\frac{n\left(A-w_{n}\right)}{(n+1) \xi},
$$

we get the corresponding $X_{n S}^{*}$ as follows:

$$
X_{n S}^{*}=\frac{\left(2 b-\frac{n+1}{n} \xi\right)+\sqrt{\left(2 b-\frac{n+1}{n} \xi\right)^{2}+3 a(A-c)}}{3 a} .
$$

2) Efficiency of the Wholesale Price Contract: As in the single-retailer case, here we define the efficiency of the wholesale price contract as

$$
R_{n}=\frac{\Pi\left(\overline{w_{n S}}\right)}{\Pi\left(\overline{w_{n}}\right)}
$$

which compares the supply chain profit with and without the wholesale price contract. Obviously $R \in[0,1)$. A lower value of $R$ indicates the wholesale price contract's impact on increasing the supply chain profit is more significant. Recall that we have equations (4), (5) and (19). So substitution and direct computation gives:

$$
\left\{\begin{aligned}
\Pi\left(\overline{w_{n}}\right)= & \frac{1}{27 a^{2}}\left(2\left(y^{2}+z\right)\left(y+\sqrt{y^{2}+z}\right)+y z\right) \\
\Pi\left(\overline{w_{n S}}\right)= & \frac{1}{27 a^{2}}\left(2\left(\left(2 b-\frac{n-2}{n} \xi\right) y_{n S}+z\right) .\right. \\
& \left(y_{n S}+\sqrt{\left.y_{n S}^{2}+z\right)}+\left(2 b-\frac{n-2}{n} \xi\right) z\right)
\end{aligned}\right.
$$

Hence

$$
\begin{aligned}
& R_{n}=\frac{\Pi\left(\overline{w_{n S}}\right)}{\Pi\left(\overline{w_{n}}\right)} \\
& =\frac{2\left(\left(2 b-\frac{n-2}{n} \xi\right) y_{n S}+z\right)\left(y_{n S}+\sqrt{y_{n S}^{2}+z}\right)+\left(2 b-\frac{n-2}{n} \xi\right) z}{2\left(y^{2}+z\right)\left(y+\sqrt{y^{2}+z}\right)+y z} .
\end{aligned}
$$

By straightforward checking we have the following proposition.

Proposition 7: The following limits hold for $R_{n}$

(a) $\lim _{\xi \rightarrow 0} R_{n}=1$ and $\lim _{n \rightarrow \infty} R_{n}=1$.

Here we exhibit some numerical examples (Fig. 3 \& Fig. 4) to show how $R$ behave under different sets of parameters.

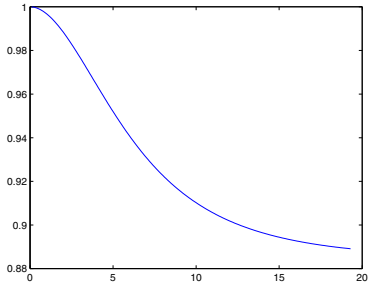

(a) $n=2$

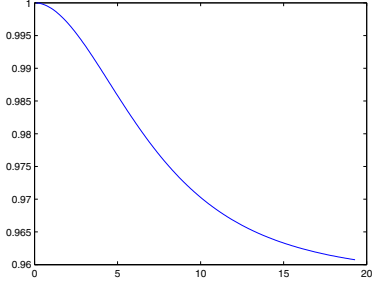

(b) $n=4$
Fig. 3. Dependence of $R$ on $\xi$ with $a=1, b=1, c=2$ and $A=40$
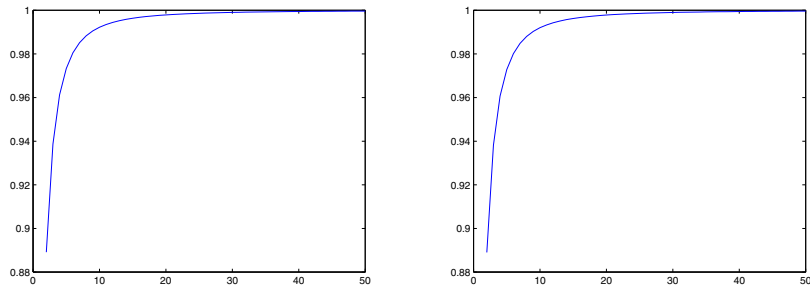

(a) $a=1, b=1, c=2, A=40 \operatorname{and}($ b) $a=5, b=3, c=3, A=40$ and $\xi=19.4$

$$
\xi=32.3
$$

Fig. 4. Dependence of $R$ on $n$

\section{B. Quantity Dependent Contract}

In this subsection, we assume that the supplier charges the $i$-th retailer $w\left(x_{i}\right)$ for per unit purchased, where the value of $w\left(x_{i}\right)$ depends on $x_{i}$, the $i$-th retailer's order level.

Proposition 8: A quantity dependent contract with

$w(x)=(1-\lambda)\left(A-\frac{\frac{n+1}{n}-2 \lambda}{2(1-\lambda)} n \xi x\right)+\lambda\left(a n^{2} x^{2}-2 b n x+c\right)$ 
is a coordination contract because given such wholesale price scheme, there is one Nash Equilibrium of the game given as follows:

$$
x_{1}^{*}=x_{2}^{*}=\ldots=x_{n}^{*}=\frac{x^{*}}{n}
$$

i.e, the aggregate order by all the retailers is the one that maximizes the supply chain profit.

Moreover, it is required that

$$
\lambda>\frac{(n-1) \xi}{2 n \sqrt{(2 b-\xi)^{2}+3 a(A-c)}} .
$$

Note that when $x_{1}^{*}=x_{2}^{*}=\ldots=x_{n}^{*}=\frac{x^{*}}{n}$, the following equality holds:

$$
\sum_{i=1}^{n} \Pi_{i}=\lambda \Pi+\frac{1-n}{2 n} \xi\left(x^{*}\right)^{2} .
$$

One can see that in this multiple-retailer case, the parameter $\lambda$ does not directly represent the proportion of total profit earned by all retailers, which is different from the single-retailer case. In fact, aggregate profit of retailers is smaller than $\lambda \Pi$ for any $n>1$. Moreover, since $(1-n) \xi\left(x^{*}\right)^{2} / 2 n$ decreases with $n$, hence with more retailers, their total profit is smaller than $\lambda \Pi$ by a larger amount.

\section{CONCluding Remarks}

In this paper, we study how to improve incentive within a supply chain by implementing two kinds of contracts, namely, wholesale price contract and quantity dependent contract. The motivation of doing so is that if supply chain coordination can be achieved given these incentives, supply chain profit can then be maximized. Since this profit represents the total wealth within the chain that is going to be split among supply chain participants, Pareto optimality is possible in this case and win-win outcome can be attained. Although supply chain coordination contracts have been extensively studied, most of the existing literature assume a fixed average total cost yet here we are considering a quadratic cost function which is able to approximate the U-shape cost curves commonly observed in various manufacturing industries and hence give results that can serve as references for a wide range of industries.

Attention is first focused on a supply chain consisting of one retailer and one supplier. We identify an optimal order level that maximizes the supply chain profit. Then it is shown that a properly designed wholesale price contract can induce the retailer to order exactly at that level and hence results in a maximal supply chain profit. However, we then demonstrate that if the supplier is entitled the right to set the wholesale price for his/her own account, actually a higher wholesale price would be preferred even though it leads to a socially suboptimal outcome. Hence, it should be expected that in order to carry out the wholesale price contract, additional transfer payment from retailer to supplier is needed to bring supplier into such contract, so extra negotiation or administration cost would occur. Thus, it motivates us to measure the efficiency of the contract to see whether such extra effort is worth or not. So we define the efficiency of the wholesale price contract $R$ to be the total profit with supplier's choice of wholesale price over the total profit with the supply chain optimal wholesale price. A lower value of $R$ means the wholesale price contract is capable of increasing the supply chain profit to a higher degree. Supply chain participants can make their decisions based on such measure. Apart from wholesale price contract, the discussion that follows is devoted to constructing a quantity dependent contract whose wholesale price is depending on the order level. It is demonstrated that there exists a quantity dependent contract which is able to coordinate the supply chain and results in a proportional devision of supply chain profit between supplier and retailer. Next the model is generalized to study a supply chain with multiple retailers and single supplier. Again we show the existence of a wholesale price contract inducing the retailers to order at an aggregate level that maximizes supply chain profit, but the supplier's interests may deviate from this wholesale price. Efficiency of the wholesale price contract in multiple-retailer case is hence defined. And then, we show that there is a quantity dependent contract leading to a Nash Equilibrium in which the retailers will make an aggregate order coincides with the supply chain profit maximizing order.

There are different possibilities for future research. For instance, we may consider modifying the contracts to deal with more complicated problems or generalizing the results to other settings.

\section{ACKNOWLEDGMENT}

Ching is supported in part by RGC Grant 7017/07P, HKU CRCG Grants, Hung Hing Ying Physical Sciences Research Fund and HKU Strategic Research Theme Fund on Computational Sciences.

\section{REFERENCES}

[1] F. Bernstein and A. Federgruen, "Decentralized supply chains with competing retailers under demand uncertainty", Manag. Sci., vol. 51, No. 1, pp. 18-29, 2005.

[2] G. P. Cachon, "Supply chain coordination with contracts", In: S. Graves, T. de Kok, eds. Handbooks in Operations Research and Management Science: Supply Chain Management, North-Holland, Amsterdam, The Netherlands, 2003.

[3] W. Ching and M. Ng, Markov Chains : Models, Algorithms and Applications, International Series on Operations Research and Management Science, Springer, New York, 2006.

[4] X. Huang, S. Choi, and W. Ching, "On improving incentive in a supply chain: wholesale price contract vs quantity dependent contract", Working paper. http://hkumath.hku.hk/ wkc/papers/papersupply.pdf.

[5] A. Marasco, "Third-party logistics: A literature review", International Journal of Production Economics, vol. 113, No. 1, pp. 127-147, 2008.

[6] A. Oke and M. Gopalakrishnan, "Managing disruptions in supply chains: A case study of a retail supply chain", International Journal of Production Economics, vol. 118, pp. 168-174, 2009.

[7] J. Spengler, "Vertical integration and anti-trust policy", Journal of Political Economy, vol. 58, pp. 347-352, 1950.

[8] A. Tsay, S. Nahmias, and N. Agrawal, "Modeling supply chain contracts: A review”, In: S. Tayur, R. Ganeshan, M. Magazine, eds. Quantitative Models for Supply Chain Management, Kluwer Academic Publishers, Dordrecht, 1999.

[9] Z. K. Weng, "Channel coordination and quantity discounts", Manag. Sci., vol. 41, No. 9, pp. 1509-1522, 1995. 